\title{
A Frame Work for Implementing Fractal Image Compression and Adaptive Byte Fractal Image Compression in Cloud Computing: A New Approach
}

\author{
Rahul Rathore \\ Department of CSE \\ Faculty of Engineering \\ TMU, Moradabad \\ Uttar Pradesh
}

\author{
Bhumika Gupta, PhD \\ Department of CSE \\ Govind Ballabh Pant Engineering \\ College \\ Pauri Garhwal, Uttarakhand
}

\author{
Kamal Kumar Gola \\ Department of CSE \\ Faculty of Engineering \\ TMU, Moradabad \\ Uttar Pradesh
}

\begin{abstract}
Cloud computing is the developing trend in technology in this present era. Cloud computing is a service oriented technology over the internet. As we know that nowadays internet is very essential in every field such as business, research etc. cloud computing delivered everything as a service either it is software, infrastructure or platform. In this research paper we propose two different frame works in which fractal image compression and adaptive byte image compression is delivered as service. Adaptive byte image compression can be delivered as a service. Adaptive byte image compression has great advantage on fractal image compression, by using adaptive byte fractal image compression the original image is compressed approximately $80 \%$ and $60 \%$ of the fractal image compression.
\end{abstract}

\section{General Terms}

Cloud Computing, Image compression, SAAS, PAAS, IASS

\section{Keywords}

Fractal Image Compression, Adaptive byte compression

\section{INTRODUCTION}

Cloud Computing sound as a solo term, but it is two separate words i.e. cloud and computing. In which cloud is a bank of services which contain services, interfaces hardware, network, and software for the end users. A service in the clouds refers as software, platform and infrastructure as a service which are delivered to client via use of internet. It enables client to perform any work on the computer or any other devices which can access internet and perform task with easy installing of any software without buying it, or can pay some amount to get access of that service. The service level agreement signed by the client and vendor before delivering the services to the client. [1].

\subsection{Deployments Models of Cloud}

There are four deployment models are recognized

\section{Private Cloud}

In this deployment model the service provider provides the services to the organization. The organization has a command over the services. The organization does not require to change their environment to access these services.

\section{Public Cloud}

This deployment model is open to all so there is a lack of security for the users, but the vendors provides the mechanism to keep information in their control for security reasons. This model is basically deployed for general public or for large companies.

\section{Community Cloud}

This deployment model is used by those organizations which have common objective. Such organizations make their own community to get the benefits of this model.

\section{Hybrid Cloud}

This deployment model has all the benefits of private, public and community model. This will beneficial for those organizations which require the facility of both private and public cloud.

\subsection{Cloud Services}

The basic services delivered by the cloud are categorized into three forms

\section{Service delivered as Software (SaaS)}

To utilize these services, the only thing is required is internet. These services are basically for commercial or general application. In this service style some software are free of cost and easy to install while others are charged on the basis of their usage.

\section{Service delivered as Platform (PaaS)}

It is mainly known as platform as a service. The aim of this service is to provide intermediate platform environment to access the service of cloud develop in different programming languages. It makes user to do not change their infrastructure such as operating system, database or storage and server to get these service.

Service delivered as Infrastructure (IaaS)

It is mainly known as Infrastructure as a service. Its aim to provide the infrastructure to provide the resources or get back the resources after their usage. The main resources used by any client are memory, working platform, network and database.

\subsection{Image Compression}

Image compression is a technique through which we can reduce the size of the image. Compression can be done in two ways i.e. lossless compression and lossy compression. Lossless image compression technique implying their name, in lossless technique the compression is done in such a manner that the decompression of image will produce the same image of the original image. There is no loss of data in lossless image compression. But in lossy technique some amount of data is lost while compressing the file. [6] 
Fractals: Fractals are geometric arrangement that can be repeated at every scale and can't be denoted by normal geometry. We can see that fractal lies in mathematical equation as well as in nature. For e.g. in nature we can see fractals in leaves in trees, mounts, lighting, veins in hand. In mathematical fractals can be seen in Julia set, Koch curve and cantor set. Fractals incalculably improve world-vision by giving a picture of much nearby us i.e. rough and small pieces of objects having structure on several dimensions like mounts, rough surfaces, coastlines etc.

\section{LITERATURE REVIEW}

In [2] the authors proposed a cloud computing frame work, with an objective to reduce the size of image over the cloud by implementing Haar Wavelet Transform as a service to save and regain the images. Then the compressed file is deployed over the cloud for effective sharing among various user.

In [3] this research work authors performed a survey on mobile cloud computing. They analyzed the issues related with cloud computing such as security, privacy, redundancy fault tolerance and provide the method to overcome from these issues.

In [4] the authors analyzed various compression techniques for digital image processing which may be lossy or lossless image compression. They identified the features and reason to adopt the correct compression technique to be used for digital image processing.

\section{PROPOSED WORK}

In this research work we are proposing two frame works of cloud computing. In first one fractal image compression is delivered as a service, which reduce the bandwidth, size of image and produce the compressed file over the cloud. And in the second one the output file (i.e. .tmp file) produced by the fractal image compression is further compressed by using adaptive byte compression algorithm, the .tmp file given as an input to adaptive byte compression algorithm, the adaptive byte compression processing the input file and produce .compressed file as final output file which is approximately $80 \%$ compressed file of the .tmp file.

\subsection{Proposed Cloud Computing Framework-I}
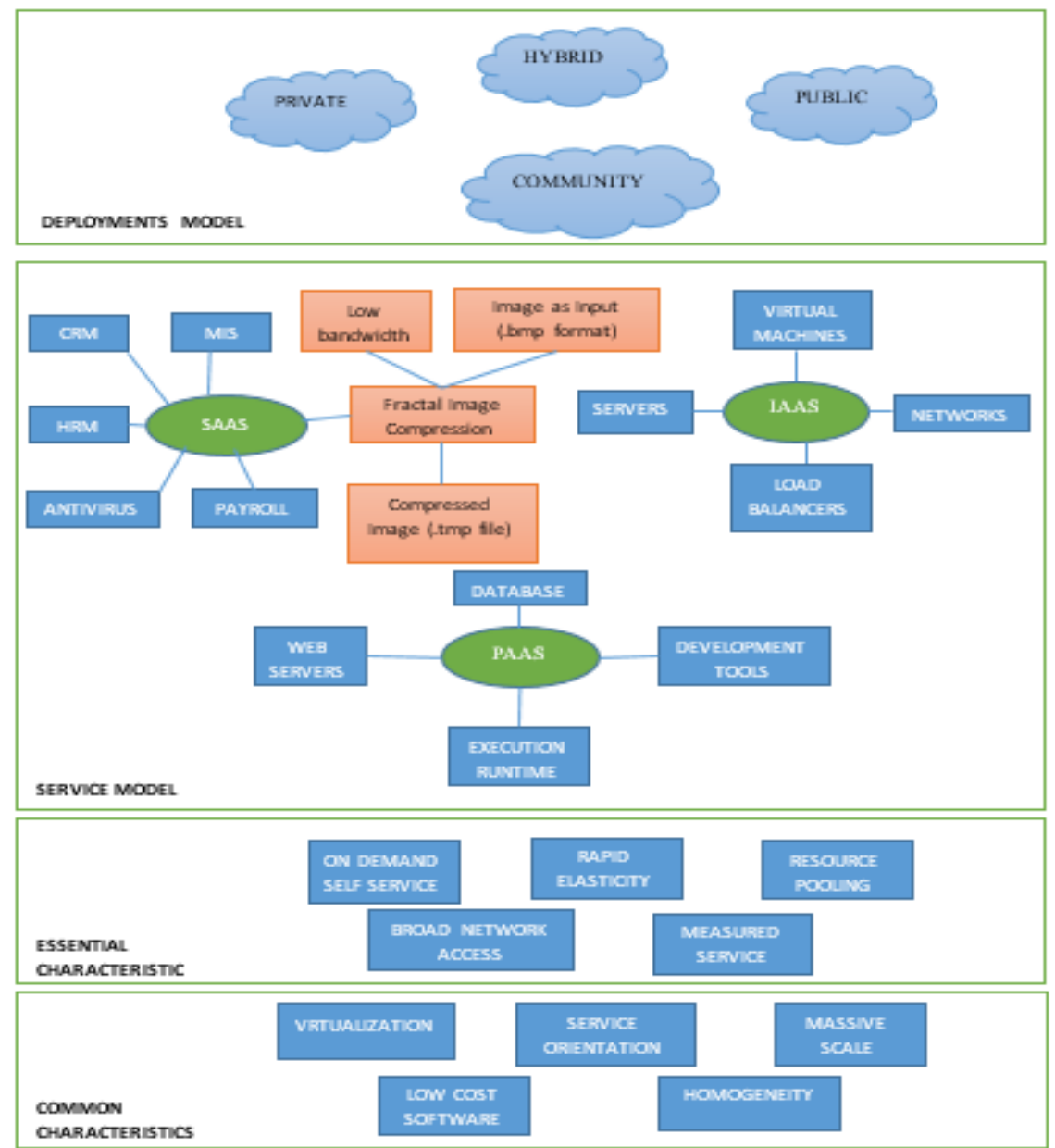

Figure. 1 Fractal image compression delivered as service 


\subsubsection{Fractal Image Compression}

FIC technique came in existence from last few years. The basic idea hidden behind image compression is to reduce redundancy and irrelevant data from the image to produce compressed file. Fractal image compression can be implemented on those images where the block of an image is identical to the real image. Fractal image compression is an efficient technique for closely coding images, in which contractive transformation is used to encode an image in which its fixed point is similar to the real image, \& then banach fixed point theorem is used to decode the encoded image. [5]. The main concept of FIC is to present an image as "iterated function system". The problem arise is that how can we effectively produce IFS for an image. A coder is used which divide an image into blocks, these blocks are coded through its own reference to rest segments of the image itself. Quad tree is the well-known method to divide the image into rectangles. An error occurred when no domain is similar to acceptable collage, for a given block. Then the given block is divided into two rectangles either vertical or horizontal cut see [7].

\subsection{Proposed Cloud Computing Framework II}

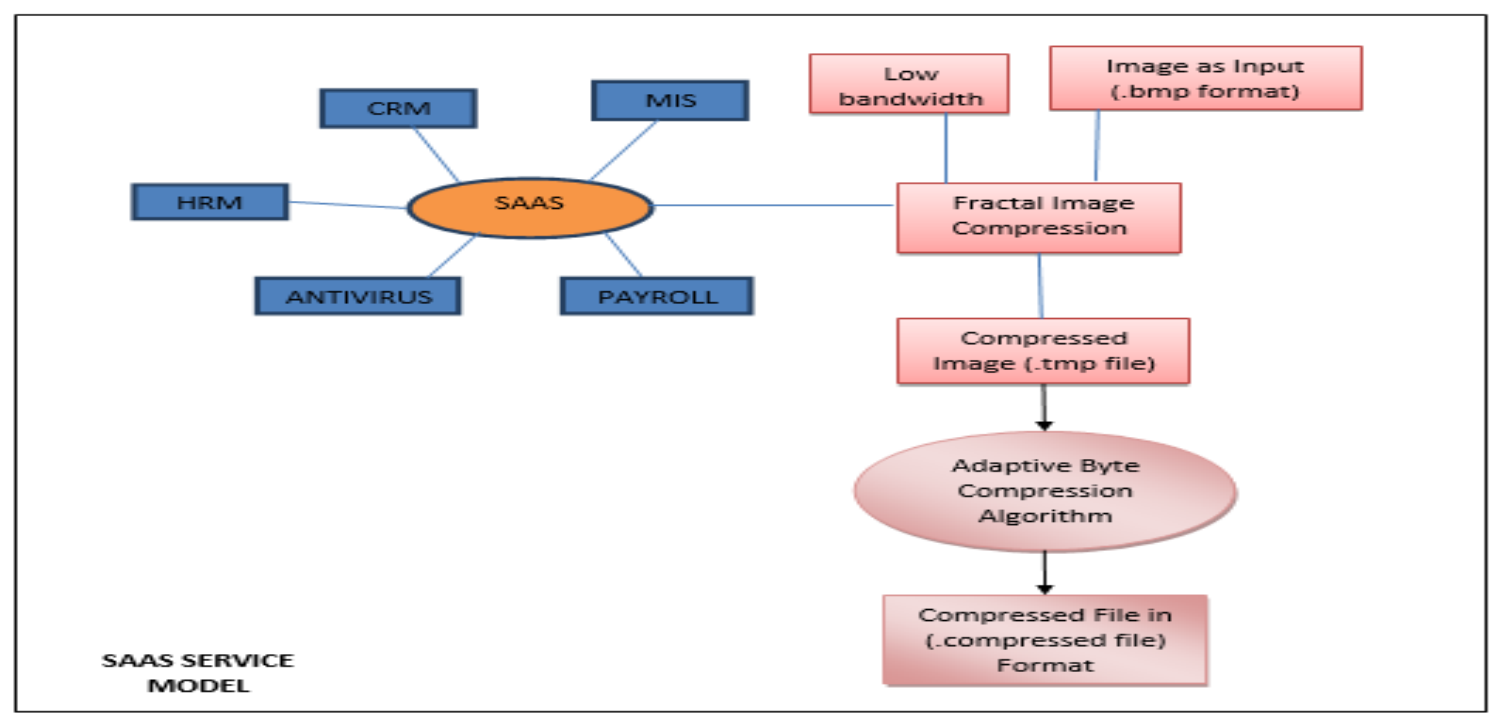

Figure. 2 Adaptive byte fractal image compression delivered as service

\subsubsection{Adaptive Byte Fractal Image Compression}

Adaptive byte compression algorithm is applied on the tmp file which is the output of fractal image compression process shown in figure 1 . This is unique algorithm which analyze the input buffer (.tmp file) and parse it. The input buffer consists of patterns of bytes. The algorithm picks the first pattern and counts the contiguous repetitions of pattern in the input buffer. It creates output buffer with the pattern at first location and followed by the count of pattern. This phenomenon is repeated throughout the input buffer taking care of buffer overflows and byte data limit overflows. Once completed for input buffer, the output buffer is created. The output buffer is called is .compressed file. When FIC is applied on an image the image is compressed to a high extend. After the application of FIC, the compression ratio is approx.30-40\% of original image but for a cloud service to work more efficiently the compression percentage has to be extened to a high extend.To achieve the above stated target, we introduce the extension of FIC as adaptive byte fractal image compression algorithm. In this the out of FIC which is in form of .tmp file is given as input to adaptive byte fractal image compression and the resultant image is called as .compressed file.

\section{RESULT AND CONCLUSION}

In this paper we are proposing a frame work in which FIC and adaptive byte image compression is delivered as service in cloud computing. By using these compression techniques, the file which is the output of adaptive byte fractal image compression is compressed approximately to $80 \%$ of the original image and compression is approximately $60 \%$ of the fractal compressed image. After comparing, fractal compression percentage and adaptive byte fractal compression we observe that the adaptive fractal compression percentage is much higher than that of fractal compression percentage this observation also states that the adaptive fractal compression percentage is enhanced multiple times than that of fractal compression percentage. The image will get compressed at higher rate and maintain the quality of image approximately as its original one. It will make easier to the end user to transmit the image at very high speed and require very low space to save it over the cloud. 


\section{REFERENCES}

[1] Rathore, Rahul. Gupta, Bhumika. Sharma, Vaibhav. Gola, Kamal Kumar: A New Approach For Load Balancing in Cloud Computing, IJCT, Vol 13., No.12, (2014)

[2] S. Girija Lakshmi, N.R. Gayathiri: A Framework For Hosting Image Compression In Cloud, IJCSMC, Vol. 3, Issue. 3, pg. 845 - 848. March(2014)

[3] Niroshinie Fernando, Seng W. Loke, Wenny Rahayu. Mobile cloud computing: A survey, Future Generation Computer Systems journal 29 84-106 (2013)

[4] Kitty Arora, Manshi Shukla. A Comprehensive Review of Image Compression Techniques, International Journal of Computer Science and Information Technologies, Vol. 5 (2), 1169-1172 (2014)

[5] C. J. Wein, E. Blake: On the performance of fractal compression with clustering, IEEE Transactions on Image Processing, 5(3), 522-526 (1996).

[6] S.Heath: "Multimedia and Communications Technology",Focal Press. pp. 120-123. ISBN 9780240515298. (1999)

[7] A. E. Jacquin: "Image Coding Based on a Fractal Theory of Iterated Contractive Image Transformations," IEEE Transactions on Image Processing, 1(1), 18-30 (1992). 